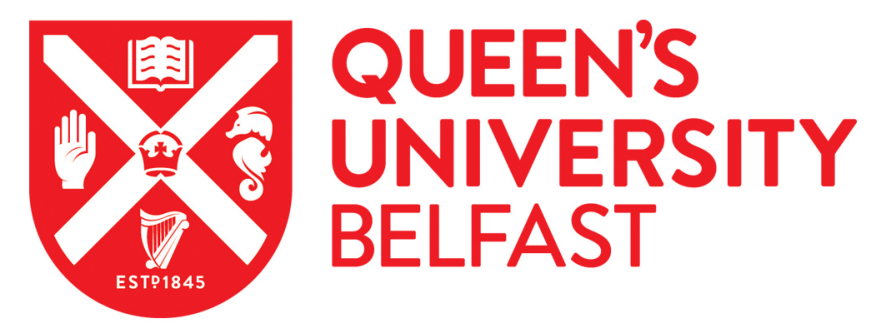

\title{
Theratyping in cystic fibrosis
}

Crawford, K. J., \& Downey, D. G. (2018). Theratyping in cystic fibrosis. Current Opinon in Pulmonary Medicine. https://doi.org/10.1097/MCP.0000000000000521

\author{
Published in: \\ Current Opinon in Pulmonary Medicine
}

Document Version:

Peer reviewed version

Queen's University Belfast - Research Portal:

Link to publication record in Queen's University Belfast Research Portal

Publisher rights

() 2018 Wolters Kluwer Health, Inc. All rights reserved.

This work is made available online in accordance with the publisher's policies. Please refer to any applicable terms of use of the publisher.

\section{General rights}

Copyright for the publications made accessible via the Queen's University Belfast Research Portal is retained by the author(s) and / or other copyright owners and it is a condition of accessing these publications that users recognise and abide by the legal requirements associated with these rights.

Take down policy

The Research Portal is Queen's institutional repository that provides access to Queen's research output. Every effort has been made to ensure that content in the Research Portal does not infringe any person's rights, or applicable UK laws. If you discover content in the Research Portal that you believe breaches copyright or violates any law, please contact openaccess@qub.ac.uk. 


\section{Theratyping in Cystic Fibrosis}

Kathryn J. Crawford ${ }^{1 *}$, Damian G. Downey ${ }^{1,2}$.

1. Northern Ireland Regional Adult Cystic Fibrosis Centre, Belfast Health and Social Care Trust, N. Ireland, UK.

2. Centre for Experimental Medicine, Queen's University Belfast, N. Ireland, UK.

*Corresponding author; kathrynj.crawford@belfasttrust.hscni.net

Address: Northern Ireland Regional Adult Cystic Fibrosis Centre, Belfast City Hospital, 51, Lisburn Road, Belfast. BT9 7AB.

Phone Number: 02890329241.

Keywords: Cystic fibrosis, CFTR modulators, theratypes, organoids. 


\section{Abstract}

\section{Purpose of review}

The treatment of cystic fibrosis (CF) with CF transmembrane conductance regulator (CFTR) modulators continues to develop at a fast pace. These compounds are potentially disease modifying but are only available to certain patient subsets based on genotype. This review discusses the role of theratyping in CF and the potential to assess all patients' response to current and emerging therapies.

\section{Recent findings}

There are limitations to treatment determined by mutation as variable clinical response to CFTR modulators has been observed within the same genotype. Patients with rare mutations not currently licensed for CFTR modulator therapy, have demonstrated response to these medications. Patient specific cellular models called organoids can be used to demonstrate response to different CFTR modulators in vitro prior to their clinical application and represent a method of theratyping.

\section{Summary}

Theratyping charts patients' clinical response to different treatments on an individual basis. This overcomes the limitations of genotype being used to predict response to individual therapies and includes all patients regardless of mutation. The use of organoids in high throughput screening allows numerous compounds to be tested on patient specific tissue pre-clinically. This could lead to the extension of theratyping beyond CFTR modulators. 


\section{Introduction}

Cystic fibrosis (CF) is a life limiting autosomal recessive condition caused by mutations in the CF transmembrane conductance regulator (CFTR) gene. The CFTR gene codes for a chloride and bicarbonate anion transporter found on the apical surface of epithelium of the respiratory, gastrointestinal and reproductive systems and over 2000 mutations have been identified to date [1,2]. Mutations lead to either non-functioning or reduced CFTR protein and therefore defective $\mathrm{Cl}^{-}$and $\mathrm{HCO}^{-}$secretion and increased $\mathrm{Na}^{+}$ absorption. This results in the production of thick, acidified secretions which in turn leads to chronic inflammation, infection, tissue death and multi organ disease [3]. Given the wide range of mutations and resulting phenotypes, this review discusses the role of theratyping in profiling patients' response to different treatments.

\section{Current CFTR classification system}

There are currently six major classes of mutations according to the primary defect in the CFTR or subsequent protein function, see table $1[3,4]$.

\begin{tabular}{|l|l|l|l|l|l|}
\hline Class I & Class II & Class III & Class IV & Class V & Class VI \\
\hline $\begin{array}{l}\text { Lack of CFTR } \\
\text { synthesis due to } \\
\text { mutations } \\
\text { generating } \\
\text { premature stop } \\
\text { codons }\end{array}$ & $\begin{array}{l}\text { CFTR protein } \\
\text { folding } \\
\text { disrupted or } \\
\text { reduced } \\
\text { preventing } \\
\text { trafficking to } \\
\text { the surface }\end{array}$ & $\begin{array}{l}\text { Lack of or } \\
\text { reduced CFTR } \\
\text { channel } \\
\text { opening at the } \\
\text { cell surface. } \\
\text { 'Gating defect' }\end{array}$ & $\begin{array}{l}\text { CFTR protein } \\
\text { structure } \\
\text { deformed } \\
\text { restricting } \\
\text { movement of } \\
\text { chloride ions } \\
\text { through the } \\
\text { pore channel- } \\
\text { 'Conductance } \\
\text { defect' }\end{array}$ & $\begin{array}{l}\text { Extreme } \\
\text { reduction in } \\
\text { production of } \\
\text { normal CFTR } \\
\text { protein, leading } \\
\text { to reduced } \\
\text { channel } \\
\text { numbers }\end{array}$ & $\begin{array}{l}\text { Accelerated } \\
\text { turnover of } \\
\text { functional } \\
\text { CFTR protein } \\
\text { at the cell } \\
\text { surface }\end{array}$ \\
\hline
\end{tabular}

Table 1. Classification system for mutations in cystic fibrosis. This table explains how different mutations in cystic fibrosis are sub divided into 6 groups according to the subsequent effects on CFTR [4].

There are limitations to the current classification system, as single mutations may have multiple class effects. F508del (c.1521_1523deICTT) has been traditionally labelled as a class II mutation [5]. However, the CFTR that does reach the apical cell surface also has defective chloride channel gating and increased channel turnover therefore displaying class III and VI features [6]. P67L (c.200C $>$ T) was initially identified as a class IV mutation, supported by a milder phenotype of pancreatic sufficiency, delayed onset of symptoms, 
but also the potential for moderate lung disease [7]. A study by Sabusap et al [8**] identified that P67L demonstrated maturation, trafficking and gating (Class II and III) defects but the absence of any conductance (class IV) defect. Additionally W1282X (c.3846G>A), a class I mutation, also possesses folding (class II) and gating defects (class III) $\left[9,10^{* *}\right]$.

A modified classification system has been produced exploring the multiplicity of CFTR mutation dysfunction. This includes the original 6 classes plus 26 of the known different combinations; however, the number of variables may limit its clinical application [10**].

CFTR modulators have the potential to be disease modifying and life prolonging. Although they represent an exciting time in the treatment of CF they have, to date, targeted specific mutations based on the classification system described above.

\section{CFTR modulator therapy}

CFTR modulators are a group of molecules, discovered by high throughput screening, that act to modify the underlying defect in the CFTR protein itself or its processing [11]. They are subdivided into potentiators, correctors or read through agents (RTAs).

Potentiators increase the flow of chloride ions through the CFTR channels by increasing their open channel probability and therefore target patients with Class III mutations [12]. Clinical trials investigating the effects of Ivacaftor (VX-770, Vertex Pharmaceuticals, Boston, Massachusetts, US) on the most common gating mutation, G551D (c.1652G>A), demonstrated an absolute 10.6\% increase in Forced Expiratory Volume in 1 second $\left(\mathrm{FEV}_{1}\right), 55 \%$ decrease in exacerbations and an improved quality of life and nutritional status [13]. The Food and Drug Administration (FDA) has approved use of Ivacaftor in a further 9 class III mutations, $\mathrm{R} 117 \mathrm{H}$ (c.350G >A) and an additional 23 residual function mutations allowing up to $10 \%$ of people with CF (PWCF) potential access to this treatment $\left[11,14^{*}\right]$.

Correctors repair defective CFTR by facilitating correct folding, processing by the cellular quality control systems and delivery to the apical membrane for functioning [15]. They are aimed at class II mutations particularly F508del. Two phase III trials, TRAFFIC and TRANSPORT, investigated the effects of LumacaftorIvacaftor (Vertex Pharmaceuticals) on patients homozygous for F508del and showed significant but modest 
absolute improvements of $3-4 \%$ in $\mathrm{FEV}_{1}[16]$. Recent trials have shown a $4 \%$ increase in $\mathrm{FEV}_{1}$ in patients homozygous for F508del in the Tezacaftor-Ivacaftor (Vertex Pharmaceuticals) group compared to the placebo group with decreased pulmonary exacerbation rates $\left[17^{*}\right]$. There are now additional pharmaceutical companies with a developing CFTR modulator pipeline that include, Flately Discovery Laboratories, Galápagos and Proteostasis Therapeutics Inc (PTI).

Early in the development of CFTR correctors it was acknowledged that a single agent had an upper limit or 'therapeutic ceiling' as to how much CFTR function could be restored [12]. However, this limit could be extended by using multiple correctors that target different defects in CFTR trafficking. Correctors can be differentiated into two different categories, C1 (such as tezacaftor and lumacaftor) which targets early folding of defective CFTR and C2, which works synergistically with the former to further augment CFTR restoration [18]. A C1 and C2 corrector combination with a potentiator forms the basis of triple therapy [18]. PTI have developed PTI-428 (Proteostasis Therapeutics, Boston, Massachusetts, US) a novel and first in class CFTR amplifier with the potential as an add-on therapy to established CFTR treatment. This mutation agnostic compound is undergoing current clinical evaluation [19].

RTAs are aimed at patients with class I mutations. These compounds allow ribosomal read through of the premature stop codons that would otherwise result in truncated, non-functional protein which are removed from the cell via nonsense mediated decay (NMD) [20,21]. This group of mutations account for 5 $10 \%$ of patients with CF [20]. Unfortunately, phase III trials of a promising RTA, Ataluren (PTC Therapeutics, South Plainfield, New Jersey, USA) failed to demonstrate clinical benefit [22]. However a new RTA, ELX-02 (Eloxx Pharmaceuticals, Waltham, Massachusetts, USA), is currently under clinical development. Treatment with this compound has demonstrated increased ion transport in fischer rat thyroid (FRT) epithelial cells transfected with $\mathrm{CFTR}^{\mathrm{G542X}}$ (c.1624G>T) or $\mathrm{CFTR}^{\mathrm{R} 1162 \mathrm{X}}$ (c.3484C>T) and Human Bronchial Epithelial (HBE) cells derived from a CF patient with a single class I mutation. Its effects are further augmented with the addition of VX-770 in these cellular models and it has also restored CFTR function in G542X transgenic mice [23].

However, variability in response to CFTR modulators even within the same mutation cohort is recognised. PWCF with rarer mutations, not explored in clinical trials, also respond to currently available CFTR 
modulators $\left[8^{* *}\right]$. A functional classification by collation of mutations based on response to current and new treatments irrespective of their current class could address some of these challenges and extend their application to all PWCF.

\section{Theratyping as a solution}

Theratyping aligns genotypes by their response to differing CFTR modulators (individually or in combination) thereby negating the challenges of using mutational class as a predictor of their efficacy $\left[10^{* *}, 24,25\right]$. This concept could expand the number of mutations treatable by a particular drug, especially for patients with rarer mutations. However, traditional randomised clinical trials (RCTs) assess drug efficacy via large standardised populations with restrictive inclusion and exclusion criteria. The absolute numbers of patients with rarer mutations would not populate a RCT and their relative exclusion from CFTR drug development programmes has resulted in considerable frustration within the CF community. Novel strategies outside the established RCT model are therefore required to test the effects of these treatments. FRT and primary nasal epithelial cells with the mutation P67L demonstrated a response to a combination of CFTR modulators, Ivacaftor and Lumacaftor [ $\left.8^{* *}\right]$. It is also likely that combinations of potentiators and correctors have some in vitro effect on class I mutations involving primary cultures of cells produced from nasal brushings from a patient homozygous for the mutation W1282X [26]. Some PWCF and residual CFTR function have increased chloride current in human nasal epithelial cell cultures and decreased sweat chloride concentration in response to Ivacaftor treatment [27]. The authors suggested that these "N-of-1" studies could be used to investigate the effect of CFTR modulators in PWCF with varying phenotypes and genotypes. Ultimately, these studies could be cohorted into umbrella and basket trials potentially speeding up access to therapy.

The 2017 FDA approval of Ivacaftor for the treatment of additional CFTR mutations was a pivotal decision based, in part, on the use of in-vitro data and not solely on RCT outcomes. This alternative approach by a regulatory body acknowledged the challenges of establishing an evidence base in a changing CF landscape [28]. 


\section{Theratyping using organoids}

Organoids are closed, three dimensional, epithelial cell structures with the apical membrane facing the internal lumen and have multiple crypt domains which contain stem cells [29]. Crypts obtained from rectal biopsies are cultured to produce intestinal organoids that serve to replicate in vivo tissue. CFTR is expressed at the apical membrane of the crypt cells allowing the passage of fluid and electrolytes into the centre of the organoid [29]. This method can produce large amounts of patient specific epithelial tissue and allows the measurement of CFTR function or can be stored for subsequent use [30].

When the c-AMP activator, forskolin, is added to intestinal organoids it produces rapid expansion due to accumulation of fluid in the lumen, which is totally dependent on CFTR function [31]. Forskolin-induced swelling (FIS) quantitatively correlates with CFTR function, as rapid FIS was observed in healthy control organoids, absent FIS in patients with CFTR null alleles, reduced FIS in milder CFTR mutations and strongly reduced FIS in organoids with severe CFTR mutations. This correlated with sweat chloride and intestinal current measurement as established biomarkers of CFTR function [31-33]. FIS is observed when CFTR function is restored in CF organoids with the addition of CFTR modulators and allows the assessment of individual drug responses [32]. FIS correlates with clinical improvements in FEV ${ }_{1}$ when assessing patients treated with CFTR modulators for the same mutation and could therefore be used to help predict in vivo response to CFTR modulators $[29,32]$. It has also been observed that there is variation in FIS amongst organoids originating from the same genotype, particularly F508del homozygotes, indicating that organoids replicate the other cellular systems determining phenotype other than just the defined CFTR mutation [34**]. Rectal biopsies are well tolerated by individuals and large amounts of tissue subsequently generated can be stored in liquid nitrogen for future testing as novel treatments become available [30,35]. However, it is recognised that intestinal epithelium may not fully represent the activity seen in the airways to these compounds $\left[34^{* *}\right]$.

A European clinical trial, HIT-CF, will obtain rectal biopsies from PWCF with rare mutations [36]. Organoid response to differing CFTR modulators will identify a patient cohort to be selected for a clinical trial of therapy. The aim is to develop 'personalised treatments' for PWCF allowing those with rare CFTR mutations access to treatment with the potential to extend to other rare genetic diseases. 
Basal cells found in the airways can form tracheospheres or bronchospheres when cultured in a 3D extracellular matrix layer [37]. These organoids consist of a layer of basal cells with differentiated ciliated and goblet cells on the luminal side. Stem cells from alveoli can also develop into alveolar spheroids capturing the properties of multiple differentiated cells present in the lower airways [38]. Nasal spheroids have been developed from tissue samples obtained from nasal curettage [39*]. Nasal epithelium cell (NEC) spheroids displayed FIS with functional CFTR. The degree of swelling correlated with CFTR correction in F508del homozygotes using Ivacaftor and lumacaftor [39*]. However, challenges arise with the narrow dynamic range of FIS between complete and absent CFTR function and the short survival of these organoids [39*]. In addition, invasive procedures are necessary to obtain the samples required and may be contaminated with multi drug resistant organisms [40].

Berical et al. [41] recently developed bronchospheres from induced pluripotent stem cells (iPSCs) developed from peripheral blood mononucleated cells from patients with cystic fibrosis with F508del and rarer genotypes. They were able to demonstrate FIS swelling in wild type bronchospheres, no swelling in F508del organoids and restoration of FIS swelling following correction of a single F508del allele [41]. Developing organoids from iPSC rather than from biopsy allows organoids to be produced without the need for invasive procedures [42]. The replicative potential of these cell types enables a large amount of epithelium to be accessed in future, as new treatments become available [43]. Limitations of this process include the complexity, expense and time consuming nature of the protocols required [43].

\section{Gene therapy and gene editing}

Gene therapy has been a focus of considerable research but has not yet resulted in a clinical therapy [44]. The aim is to safely and efficiently deliver CFTR cDNA to the airway epithelium to allow CFTR expression and has the potential to be a future treatment [45]. Challenges include finding suitable vectors (either viral or non-viral), reducing the immunogenicity of treatment and achieving adequate CFTR gene expression in the lung [44]. A phase IIb trial assessing the clinical efficacy of CFTR gene-liposome complex pGM169/GL67A against $0.9 \%$ saline (placebo) showed a modest but significant $3.7 \%$ increase in $\mathrm{FEV}_{1}$ 
compared with placebo at 12 months. This however, represented a stabilisation in $\mathrm{FEV}_{1}$ within the treatment group rather than an improvement [46].

Gene editing can also be used to correct mutations in the CFTR gene by repairing the underlying DNA. CRISPR/Cas9 technology consists of guide RNA (gRNA) to identify specific nucleotide sequences and an endonuclease (Cas9) which cleaves DNA to enable gene editing [47]. Intestinal organoids carrying F508del have demonstrated FIS, similar to non CF organoids, following correction by CRISPR/Cas9 compared with cells that have not undergone repair [48]. Gene editing has therefore the potential as a future therapy but challenges remains with the methodology and frequency of delivery.

\section{Conclusions}

Theratyping is a method of profiling patients according to their response to current and potential future therapies. The aim is to include all PWCF in testing and therefore access to medications for all who could benefit. This could avoid the challenges of variable response to CFTR modulators within the same genotype and allows access to potentially disease modifying treatment for those with rare genotypes. Organoids provide a pre-clinical platform to theratyping by assessing response to a range of CFTR modulators. Although the application of intestinal organoids is at a relatively advanced stage of development, the in vitro response may not fully correlate with clinical outcomes. Airway organoids are a potential solution to this issue, but their development is still in a proof-of-concept stage. Further studies are required to determine the threshold of organoid swelling that correlates with meaningful clinical outcomes to the patient. The future of theratyping could expand beyond response to CFTR modulators and explore epigenetic, inflammatory and microbiome profiling leading to a multifaceted, personalised approach to treatment for PWCF.

\section{Key points}

- CF is caused by mutations in the CFTR gene and are currently grouped into six classes according to the presumed resulting defect in CFTR protein.

- There are limitations to this classification system, as mutations can have multiple class effects. 
- There are varying responses to CFTR modulators within the same genotype and patients with rare mutations do not have access to these therapies.

- Theratyping assesses patients' response to treatments regardless of mutation.

- Organoids can be used as a guide to clinical response to CFTR modulators and ensure all patients have potential access to disease modifying treatment.

Acknowledgements: None declared.

\section{References}

1. Elborn SJ. Cystic fibrosis. Lancet 2016; 388:2519-2531.

2. US CF Foundation, John Hopkins University, Hospital for Sick Children. CFTR2. Clinical and functional translation of CFTR. response . https://www.cftr2.org/resources. Accessed on 11th May 2018.

3. Rowe SM, Miller S, Sorscher EJ. Cystic fibrosis. N Engl J Med 2005; 352:1992-2001.

4. Addy C, Downey DG, Elborn JS. Improvements in symptomatic treatment strategies for cystic fibrosis: delivering CF care in the 21st century. Expert Opinion on Orphan Drugs 2015; 4(1):5-19.

5. Cheng SH, Gregory RJ, Marshall J, et al. Defective intracellular transport and processing of CFTR is the molecular basis of most cystic fibrosis. Cell 1990; 63(4):827-834.

6. Dalemans W, Barbry P, Champigny G, et al. Altered chloride ion channel kinetics associated with the delta F508 cystic fibrosis mutation. Nature 1991; 354(6354):526-528. 
7. Gilfillan A, Warner JP, Kirk JM et al. P67L: a cystic fibrosis allele with mild effects found at high frequency in the Scottish population. J Med Genet 1998; 35(2):122-125.

8. **Sabusap CM, Wang W, McNicholas CM et al. Analysis of cystic fibrosis-associated P67L CFTR illustrates barriers to personalized therapeutics for orphan diseases. JCI insight 2016; 1(14):e86581.

Research article demonstrating that P67L has multiple mutational class effects and that it shows in vitro response to CFTR modulators.

9. Xue X, Mutyam V, Tang L et al. Synthetic aminoglycosides efficiently suppress cystic fibrosis transmembrane conductance regulator non sense mutations and are enhanced by ivacaftor. Am J Respir Cell Mol Biol 2014; 50:805-816.

10. **Vite G, Radu G, Avramescu et al. From CFTR biology toward combinatorial pharmacotherapy: expanded classification of cystic fibrosis mutations. Mol Biol Cell 2016; 27(3):424-432.

This article explores how CFTR mutations have multiple class effects and that a new classification system could help direct selection of therapy.

11. Ong T, Ramsey BW. New Therapeutic Approaches to Modulate and Correct Cystic Fibrosis Transmembrane Conductance Regulator. Pediatr Clin of North Am 2016; 63(4):751-764.

12. Van Goor F, Straley KS, Cao D, et al. Rescue of DeltaF508-CFTR trafficking and gating in human cystic fibrosis airway primary cultures by small molecules. Am J Physiol Lung Cell Mol Physiol 2006; 290(6):L11171130.

13. Ramsey BW, Davies J, McElvaney NG, et al. A CFTR potentiator in patients with cystic fibrosis and the G551D mutation. N Engl J Med 2011; 365:1663-1672. 
14. *Burgener EB, Moss RB. Cystic Fibrosis transmembrane conductance regulator modulators: precision medicine in cystic fibrosis. Curr Opin Pediatr 2018; 30(3):372-377.

This review summarises the current use of CFTR modulators in CF and ongoing developments with this therapy.

15. Rowe SM, Verkman AS. Cystic fibrosis transmembrane regulator correctors and regulators. Cold Spring Harb Perspect Med 2013; 3(7):a009761.

16. Wainwright CE, Elborn JS, Ramsey BW, et al. Lumacaftor-Ivacaftor in Patients with Cystic Fibrosis Homozygous for Phe508del CFTR. N Engl J Med 2015; 373(3):220-231.

17. *Taylor-Cousar JL, Munck A, McKone EF, et al. Tezacaftor-Ivacaftor in Patients with Cystic Fibrosis Homozygous for Phe508del. N Engl J Med 2017; 377(21):2013-2023.

This article presents the randomised control trial (RCT) data showing that the combination of tezacaftorivacaftor is safe and efficacious in patients 12 years or older homozygous for F508del.

18. * Li H, Pesce E, Sheppard DN, et al. Therapeutic approaches to CFTR dysfunction: From drug discovery to drug development. J Cyst Fibros 2018; 17(2):S14-S21.

This article discusses the multiple effects that CF mutations have on CFTR protein and therefore several approaches are required to restore its function. Areas covered include CFTR modulators and potential future therapies such as proteostasis regulators and anion transporters.

19. Flume P, Sawicki G, Pressler T et al. Phase 2 initial results evaluating PTI-428, a novel CFTR amplifier, in patients with cystic fibrosis. J Cyst Fibros 2018: 17(S3): S1-2.

20. Oren YS, Pranke IM, Kerem B, Sermet-Gaudelus I. The suppression of premature termination codons and the repair of splicing mutations in CFTR. Curr Opin in Pharmacol, 2017; 34:125-131. 
21. Kellermayer R. Translational readthrough induction of pathogenic nonsense mutations. Eur J Med Genet 2006; 49(6):445-450.

22. PTC therapeutics. PTC Therapeutics Announces Results from Pivotal Phase 3 Clinical Trial of Ataluren in Patients Living with Nonsense Mutation Cystic Fibrosis. http://ir.ptcbio.com/news-releases/news-releasedetails/ptc-therapeutics-announces-results-pivotal-phase-3-clinical Accessed on 11th May 2018.

23. Rowe SM, Mutyam V, Alroy I, et al. Translational read-through of CFTR nonsense mutations and inducement of cystic fibrosis transmembrane conductance regulator (CFTR) function by ELX-02 treatment. J Cyst Fibros. 2018: 17(S3):S2.

24. Milla CE, Moss RB. Recent advances in cystic fibrosis. Curr Opin Pediatr 2015; 27(3):317-324.

25. Cutting GR. Cystic fibrosis genetics: from molecular understanding to clinical application. Nat Rev Genet 2015; 16(1):45-56.

26. Haggie PM, Phuan PW, Tan JA, et al. Correctors and potentiators rescue function of the truncated W1282X-cystic fibrosis transmembrane regulator (CFTR) translation product. J Biol Chem 2017; 292(3):77185.

27. McGarry ME, Illek B, Ly NP, et al. In vivo and in vitro ivacaftor response in cystic fibrosis patients with residual CFTR function: N-of-1 studies. Ped pulm 2017; 52: 472-479.

28. Durmowicz AG, Lim R, Rogers H, et al. The U.S. Food and Drug Administration's experience with Ivacaftor in Cystic Fibrosis. Establishing Efficacy using In vitro data in Lieu of a Clinical Trial. Ann of Am Thorac Soc 2018; 15:1-2. 
29. Noordhoek J, Gulmans V, van der Ent K, Beekman JM. Intestinal organoids and personalized medicine in cystic fibrosis: a successful patient-orinetated research collaboration. Curr Opin Pulm Med 2016; 22:610616.

30. Sato T, Clevers H. Growing self organizing mini-guts from a single intestinal stem cell: mechanism and applications. Science 2013; 340:1190-1194.

31. Dekkers JF, Wiegerinck $\mathrm{CL}$, de Jonge $\mathrm{HR}$, et al. A functional CFTR assay using primary cystic fibrosis intestinal organoids. Nat Med 2013; 19(7):939-945.

32. Dekkers JF, van der Ent CK, Beekman JM. Novel opportunities for CFTR-targeting drug development using organoids. Rare Diseases 2013; 1:e27112.

33. Zomer-van Ommen DD, de Poel E, Kruisselbrink E et al. Comparison of ex vivo and in vitro intestinal cystic fibrosis models to measure CFTR-dependent ion channel activity. J Cyst Fibros 2018; 17:316-24.

34. ${ }^{* *}$ Dekkers JF, Berkers G, Kruisselbrink E, et al. Characterizing responses to CFTR-modulating drugs using rectal organoids derived from subjects with cystic fibrosis. Sci Transl Med 2016; 8(344):1-12. This research article showed how in vitro drug responses in rectal organoids positively correlated with published data from clinical trials with VX-809 and VX-770. They suggest that rectal organoids could be used to predict which patients would benefit from CFTR modifying treatment.

35. Sevidoni MF, Sousa M, Vinagre AM et al. Rectal forceps biopsy procedure in cystic fibrosis: technical aspects and patients perspective for clinical trials feasibility. BMC Gastroenterol 2013; 13:91. 
36. HIT-CF Europe: For doctors. Available from https://www.hitcf.org/for-doctors/. Accessed on 14th May 2018.

37. Rock JR, Onaitis MW, Rawlins EL, et al. Basal cells as stem cells of the mouse trachea and human airway epithelium. Proc. Natl. Acad. Sci. USA 2009; 106:12771-12775.

38. Barkauskas CE, Cronce MJ, Rackley CR, et al. Type 2 alveolar cells are stem cells in adult lung. J Clin Invest 2013; 123(7):3025-3036.

39. *Brewington JJ, Filbrandt ET, LaRosa FJ 3rd, et al. Detection of CFTR function and modulation in primary nasal cell spheroids. J Cyst Fibros 2018; 17:26-33.

This article describes how nasal spheroids can used to improve understanding of rare CFTR mutations and their response to CFTR modulator therapy.

40. Randell SH, Walstad DL, Schwab UE, et al. Isolation and culture of airway epithelial cells from chronically infected human lungs. In Vitro Cell Dev Biol Anim 2001; 37:480-489.

41. Berical A, McCauley KB, Thomas DC, et al. A2463 - Induced Pluripotent Stem Cell-Derived Bronchospheres as a Novel Platform for Personalized Cystic Fibrosis Drug Prediction. ATS International Conference, San Diego 20 $0^{\text {th }}$ May 2018. Accessed from http://www.abstractsonline.com/pp8/\#!/4499/presentation/16202 on 28th May 2018.

42. Loh YH, Agarwal S, Park IH et al. Generation of induced pluripotent stem cells from human blood. Blood 2009; 113(22):5476-5479.

43. Mou H, Brazausksa K, Rajagopal J. Personalized medicine for cystic fibrosis: Establishing human model systems. Pediatric Pulmon 2015; 50:S14-S23. 
44. Hart SL, Harrison PT. Genetic therapies for cystic fibrosis lung disease. Current opinion in pharmacology $2017 ; 34: 119-24$

45. Sinn PL, Anthony RM, McCray Jr PB. Genetic therapies for cystic fibrosis lung disease. Human molecular genetics 2011; 20(R1):R79-86.

46. Alton EW, Armstrong DK, Ashby D et al. Repeated nebulisation of non-viral CFTR gene therapy in patients with cystic fibrosis: a randomised, double-blind, placebo-controlled, phase $2 b$ trial. Lancet Resp Med 2015; 3:684-91.47. Jiang F, Doudna JA. CRISPR-Cas9 structures and mechanisms. Ann Rev Biophys 2017; 46:505-529.

48. Schwank G, Koo BK, Sassellin V, et al. Functional repair of CFTR by CRISPR/Cas9 in intestinal stem cell organoids of cystic fibrosis patients. Cell Stem Cell 2013; 13(6):653-658. 